\title{
Expressão das Proteínas p53 E Cox-2 em Adenocarcinoma Intestinal e Mucosa Adjacente
}

\author{
Expression of p53 and Cox-2 Proteins in Intestinal \\ Adenocarcinoma and Adjacent Mucosa
}

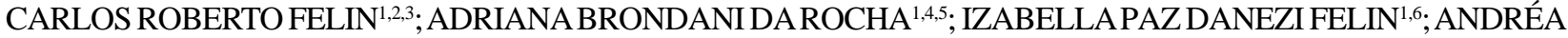 \\ REGNER $^{1,4,5}$; IVANAGRIVICICH ${ }^{1,4,5}$
}

\begin{abstract}
${ }^{1}$ Programa de Pós Graduação em Genética e Toxicologia Aplicada, Universidade Luterana do Brasil, Canoas, RS, Brasil; ${ }^{2}$ Hospital da Brigada Militar de Santa Maria, RS, Brasil; ${ }^{3}$ Hospital Universitário da Universidade Federal de Santa Maria, Santa Maria, RS, Brasil; ${ }^{4}$ Programa de Pós Graduação em Diagnóstico Genético e Molecular, Universidade Luterana do Brasil, Canoas, RS, Brasil; ${ }^{5}$ Laboratório de Marcadores de Estresse Celular, Centro de Pesquisas em Ciências Médicas, Universidade Luterana do Brasil, Canoas, RS, Brasil; ${ }^{6}$ Departamento de Patologia, Universidade Federal de Santa Maria, RS, Brasil.
\end{abstract}

FELIN CR; ROCHA AB; FELIN IPD; REGNER A; GRIVICICH I. Expressão das Proteínas p53 e Cox-2 em Adenocarcinoma Intestinal e Mucosa Adjacente. Rev bras Coloproct, 2008;28(1): 019-025.

RESUMO: Este estudo teve como objetivo verificar se existe associação entre o estadiamento do tumor e a expressão das proteínas p53 e Ciclooxigenase-2 (Cox-2) em adenocarcinoma de intestino. Foi realizado um estudo retrospectivo de 40 blocos de parafina contendo amostras obtidas de ressecção cirúrgica de tecido intestinal anteriormente diagnosticado como adenocarcinoma de intestino humano. O material foi coletado no período entre 1998 e 2003 no Hospital Universitário de Santa Maria, RS, Brasil. Como controle, foram utilizadas amostras de áreas não tumorais de mucosa adjacente, referentes a cada caso $(n=40)$. Utilizamos a imuno-histoquímica para analisar este material quanto à expressão das proteínas p53 e Cox-2. A expressão das proteínas p53 e Cox-2 foi significativamente maior em tecido tumoral quando comparada com mucosa adjacente. Detectamos positividade total para Cox-2 (100\%), e parcial (70\%) para p53 em tecido tumoral. Foi verificada associação significativa entre expressão da proteína p53 e estadiamento segundo Astler-Coller, mas não com a classificação TNM. Quanto à proteína Cox-2, não foi observada associação significativa com estadiamento do tumor de intestino. Os achados sugerem que existe uma tendência entre a expressão da proteína p53 e Cox-2 com o estadiamento do tumor, sendo necessária comprovação com estudos futuros.

Descritores: Adenocarcinoma de intestino. Proteína p53. Proteína Cox-2. Imuno-histoquímica. Estadiamento tumoral.

\section{INTRODUÇÃo}

O carcinoma de intestino é um dos tumores malignos mais comuns no ocidente, correspondendo a terceira causa de morte por câncer ${ }^{1}$. Mais de $80 \%$ desses tumores são esporádicos, sendo que os fatores ambientais e dietéticos parecem ser variáveis de grande valor no desenvolvimento desta neoplasia. A história familiar para o câncer intestinal é observada em até $25 \%$ dos casos, e algumas situações como presença de adenomas, constituem risco de desenvolver adenocarcinoma intestinal ${ }^{2,3,4}$.
O diagnóstico do câncer colorretal pode ser suspeitado pela história clínica de enterorragia ou sangue oculto nas fezes, dor abdominal, emagrecimento, e confirmado pela endoscopia baixa seguida de biópsia e anatomopatológico ${ }^{1}$. Existem fatores prognósticos para o câncer colônico que estão diretamente relacionados ao sistema pTNM de estadiamento ${ }^{5}$. São estes: infiltração do tumor na gordura pericólica, graduação histológica, obstrução, perfuração, aderência do tumor a órgãos e estruturas adjacentes, invasão angiolinfática e classificação de Dukes e Astler-Coller ${ }^{3,4,6}$. O prognóstico em casos de doença avançada é ruim, e mais

Trabalho realizado no Laboratório de Marcadores de Estresse Celular, Centro de Pesquisa em Ciências Médicas, Universidade Luterana do Brasil - Rio Grande do Sul - Brasil. 
de um terço dos pacientes morrem durante a progressão da doença ${ }^{7}$.

Durante a evolução dos tumores de intestino, do tipo esporádico, vários genes são alterados, sendo os mais freqüentes: TP53, $k$-ras, DCC, $A P C$ e $c$ $m y c^{3,4,8,9}$. Atualmente vários marcadores imunohistoquímicos vêm sendo estudados em relação à carcinogênese colorretal, entre eles a oncoproteína p53 e, mais recentemente, a Ciclooxigenase- $2^{10,11}$.

Quando ocorre dano ao DNA, o gene TP53 selvagem inibe a mitose e induz o reparo, se este não for eficiente, ele desencadeia apoptose. Assim, células geneticamente instáveis e com predisposição a transformação maligna são eliminadas. Entretanto, quando ocorre uma mutação no gene TP53, há alteração funcional da proteína p53 que se torna não funcional, levando a uma instabilidade genética e iniciando a transformação maligna ${ }^{12}$. A mutação do TP53 proporciona acúmulo dessa proteína no núcleo celular, pois a alteração genética determina uma meia vida mais longa, podendo ser detectada por imuno-histoquímica ${ }^{13}$.

A ciclooxigenase (Cox) é uma enzima limitante da taxa de conversão do ácido aracdônico em prostaglandina ${ }^{10}$. Esta enzima possui duas isoformas a Cox-1 e a Cox-2. A Cox-1 é expressa constitutivamente na maioria dos tecidos, e controla atividades fisiológicas normais. Já a Cox-2 somente se expressa após ser estimulada por fatores de crescimento, citoquinas e mitógenos. A Cox-2 é expressa no trato gastrointestinal em níveis indetectáveis, porém sua expressão é evidenciada na maioria dos adenomas e eleva-se significativamente no carcinoma intestinal ${ }^{1,14,15,16}$. A expressão desta enzima pode ser um fator prognóstico independente, cujo nível de expressão tem sido relacionado ao desenvolvimento de metástases à distância, recorrências, menor sobrevida e grau de invasão vascular ${ }^{10}$.

Neste estudo, avaliamos se existe associação entre a expressão das proteínas p53 e Cox-2 com o estadiamento do adenocarcinoma intestinal.

\section{MÉTODOS}

\section{Seleção de casos}

Foram escolhidos blocos de parafina $(n=40)$ contendo fragmentos de tecido intestinal anteriormente diagnosticado como adenocarcinoma de intestino humano. Todos provenientes do arquivo do Serviço de Patologia do Hospital Universitário de Santa Maria (HUSM), RS, Brasil, referentes aos exames histopatológicos rotineiros realizados no período entre 1998 e 2003. Para controle, amostras de áreas não tumorais de mucosa adjacente, referentes a cada caso, foram incluídas no estudo $(\mathrm{n}=40)$.

Como critério de inclusão consideramos a existência e a conservação de todos os blocos de parafina referentes a cada caso no período de tempo destinado ao estudo. Foram considerados critérios de exclusão a má conservação dos blocos de parafina, arquivo incompleto, dúvidas diagnósticas, outros diagnósticos, produtos de biópsias e casos fora do período estipulado.

O protocolo experimental foi submetido e aprovado pelo Comitê de Ética em Pesquisa em Seres Humanos e Animais da Universidade Luterana do Brasil , Canoas, RS, Brasil (protocolo CEP-ULBRA 2006-256H).

\section{Processamento histológico}

Foram obtidos de todos os blocos cortes histológicos com $4 \varnothing \mathrm{m}$ de espessura, montados em lâminas histológicas tratadas com silano (4\%) e coradas com hematoxilina e eosina para reavaliação histopatológica.

A revisão histopatológica em todos os casos foi realizada por dois patologistas experientes, de forma independente, sem identificação dos casos. Eventuais resultados conflitantes foram discutidos pelos mesmos para definição consensual da análise.

Procedeu-se graduação, estadiamento pTNM e caracterização de itens relativos ao preenchimento de um instrumento recomendado para adenocarcinoma coloretal $^{17}$, adotado pelo Serviço de Patologia do HUSM.

\section{Imuno-histoquímica}

Utilizando a técnica de imuno-histoquímica, foram testadas, em todos os casos de adenocarcinoma de intestino e mucosa adjacente, a imunorreatividade da proteína p53 utilizando o sistema Envison ${ }^{\mathrm{TM}}$ (Dako, EUA), e para a proteína Cox-2, foi utilizado o método da avidina-biotina-peroxidase (ABC) (Vector Laboratories, EUA) com modificações ${ }^{8}$.

As lâminas contendo tecido intestinal foram desparafinizadas em xilol, hidratadas em álcool etílico absoluto, tratadas com tampão citrato $(\mathrm{pH} 6,0)$ para a reativação do antígeno e lavadas em solução salina tamponada. Após, as lâminas foram tratadas com peróxido de hidrogênio $\left(\mathrm{H}_{2} \mathrm{O}_{2}\right)$ a $3 \%$ por cinco minutos, para bloqueio da peroxidase endógena. A seguir os tecidos foram incubados com os respectivos anticorpos primários p53 (anticorpo monoclonal de camundongo, clone D07, 
DAKO-EUA, 1:1800) e Cox-2 (anticorpo monoclonal de camundongo, Cayman Chemical Company-EUA; 1:100), overnight a $4^{\circ} \mathrm{C}$. Posteriormente, os cortes foram incubados com o anticorpo secundário biotinilado por $60 \mathrm{mi}$ nutos, e durante 45 minutos, no caso do sistema Envision ${ }^{\mathrm{TM}}$.

A revelação dos anticorpos foi realizada através da reação da enzima peroxidase visualizada pela coloração com DAB ((3-3)-tetrahidrocloreto de diaminabenzidina), contra-corados com hematoxilina de Harris e analisados em microscópio óptico.

\section{Análise imuno-histoquímica}

A imunocoloração da proteína p53 foi considerada positiva quando observamos mais de $10 \%$ de células imunomarcadas com reatividade nuclear para p53. O controle positivo para a imunolocalização da proteína p53 foi feito com uma amostra de carcinoma de mama. Controles negativos foram obtidos pela omissão dos anticorpos primários ${ }^{14,19}$.

Os critérios utilizados para avaliação da Cox-2 foram: Negativo = ausência de reatividade; Positivo $1+=$ reatividade fraca; Positivo $2+=$ reatividade moderada e Positivo $3+=$ reatividade intensa. Foi utilizado como controle positivo uma amostra de carcinoma de mama com reatividade intensa a Cox-2 (3+).

\section{Análise estatística}

A análise estatística dos resultados da imunorreatividade das proteínas p53 e Cox-2 e sua associação com o estadiamento do tumor foi obtida com o emprego do teste qui-quadrado de associação. Para a comparação da expressão das proteínas entre o tecido normal e o tecido tumoral empregou-se o teste exato de Fischer. Para todos os testes foi utilizado o software GraphPad Instat (versão 3.05, GraphPad Software Incorporation, San Diega, CA, USA).

\section{RESULTADOS}

\section{Características da amostra de adenocar- cinoma de intestino}

Foram estudados 40 casos de adenocarcinoma de intestino, 28 (70 \%) em mulheres e $12(30 \%) \mathrm{em}$ homens, predominantemente dos 61 aos 80 anos (55 \%). Com relação à localização do tumor houve predomínio do cólon descendente $(\mathrm{n}=38 \%)$.

A maioria dos tumores correspondeu a adenocarcinoma moderadamente $(\mathrm{n}=26 ; 65 \%)$ ou bem diferenciados $(n=23 ; 32 \%)$. Em $75 \%$ da amostra a avaliação da invasão vascular foi ausente; e 92,5 \% dos casos não apresentaram invasão perineural (Tabela 1).

Tabela 1 - Características gerais das amostras de adenocarcinoma de intestino.

\begin{tabular}{|c|c|}
\hline Característica & $\mathbf{N}^{0} \operatorname{casos}$ \\
\hline Sexo Masculino & 12 \\
\hline Feminino & 28 \\
\hline Idade $<50$ & 7 \\
\hline $51-60$ & 8 \\
\hline $61-70$ & 11 \\
\hline $71-80$ & 11 \\
\hline$>80$ & 3 \\
\hline \\
\hline \multicolumn{2}{|l|}{$\begin{array}{l}\text { Ângulo Hepático } \\
\text { Ân }\end{array}$} \\
\hline Cólon Descendente & 38 \\
\hline \multirow{2}{*}{\multicolumn{2}{|c|}{$\begin{array}{l}\text { Cólon Ascendente } \\
\text { Grau de Diferenciação }\end{array}$}} \\
\hline & \\
\hline \multicolumn{2}{|l|}{ Bem Diferenciado } \\
\hline Moderadamente Diferenciado & 26 \\
\hline Pouco Diferenciado & 1 \\
\hline \multicolumn{2}{|l|}{ Invasão Vascular } \\
\hline Presente & 10 \\
\hline & 30 \\
\hline \multicolumn{2}{|l|}{ Invasão Perineural } \\
\hline Presente & 3 \\
\hline Ausente & 37 \\
\hline \multicolumn{2}{|l|}{ Estadiamento } \\
\hline I & 7 \\
\hline IIA & 8 \\
\hline IIB & 4 \\
\hline IIIA & 2 \\
\hline IIIB & 5 \\
\hline IIIC & 11 \\
\hline IV & 3 \\
\hline \multicolumn{2}{|l|}{ Classificação de Dukes } \\
\hline A & 7 \\
\hline B & 12 \\
\hline $\mathrm{C}$ & 18 \\
\hline $\mathrm{D}$ & 3 \\
\hline \multicolumn{2}{|l|}{ Classificação de Astler-Coler } \\
\hline $\mathrm{B} 1$ & 7 \\
\hline B2 & 12 \\
\hline $\mathrm{C} 1$ & 3 \\
\hline $\mathrm{C} 2$ & 15 \\
\hline $\mathrm{D}$ & 3 \\
\hline
\end{tabular}


Em relação ao estadiamento tumoral os resultados apontam predomínio dos estágios IIIC em 11 casos $(27,5 \%)$ e IIA em 8 casos (20 \%) (Tabela 1). A classificação de Dukes evidenciou predominância da amostra nos estágios Dukes C e B; sendo 18 casos (45 \%) Dukes C e 12 casos (30 \%) Dukes B (Tabela 1). A classificação de Astler-Coler mostrou predominância nos estágios Astler-Coler C2 em 15 casos $(37,5 \%)$ e Astler-Coler B2 em 12 casos (30\%) (Tabela 1).

\section{Expressão imuno-histoquímica de p53 e} Cox-2 em tecido normal e tumoral

Foi observada hiperexpressão de p53 em 28 das 40 amostras de adenocarcinoma $(70 \%)$ e reatividade Cox-2 3+ em $100 \%$ dos casos, representando diferença estatisticamente significante do tecido normal ( $\mathrm{p}<0.0001)$ em relação aos marcadores p53 e Cox-2 (Tabela 2).

Associação entre estadiamento segundo TNM e Astler-Coller com marcadores p53 e Cox-2
Não foi observada diferença significativa ao considerarmos a associação entre hiperexpressão de p53 e estadiamento segundo TNM, apesar da maioria $(27,5 \%)$ ser classificada como estágio IIIC (Tabela 3). Quinze casos (37,5\%) dos adenocarcinomas com hiperexpressão para p53, encontravam-se no estágio C2, segundo Astler-Coller, representando associação estatística significativa entre p53 e estadiamento segundo Astler-Coller $(\mathrm{p}=0.0244)(\mathrm{Ta}-$ bela 3 ).

Em relação ao Cox-2 positivo $3+$, não observamos diferença significativa ao considerarmos a associação desta proteína com estadiamento TNM ou Astler-Coller (Tabela 4).

\section{DISCUSSÃO}

O conhecimento do maior número de variáveis diretamente associadas ao prognóstico dos tumores intestinais torna-se necessário para a melhor identificação de pacientes que poderão se beneficiar

Tabela 2 - Reatividade imuno-histoquímica da p53 e Cox-2 nas amostras de adenocarcinoma de intestino e tecido normal.

\begin{tabular}{llrrrrr}
\hline & (n) & \multicolumn{3}{c}{ p53* $^{*}$} & \multicolumn{3}{c}{ Cox-2* } \\
\hline & & + & - & +1 & +2 & +3 \\
Tecido normal & 40 & 0 & 40 & 5 & 26 & 9 \\
Tecido tumoral & 40 & 28 & 12 & 0 & 0 & 40 \\
\hline
\end{tabular}

* Estatisticamente diferente do tecido normal $(p<0.0001)$.

Tabela 3 - Associação entre o estadiamento segundo sistema TNM e Astler-Coller no adenocarcinoma de intestino e a expressão da proteína p53.

\begin{tabular}{|c|c|c|c|c|c|c|c|c|}
\hline \multicolumn{9}{|c|}{ Estadiamento } \\
\hline \multirow[t]{2}{*}{ p53 } & & \multicolumn{6}{|c|}{ TNM } & \multirow[t]{2}{*}{ Total } \\
\hline & I & IIA & IIB & IIIA & IIIB & IIIC & IV & \\
\hline+ & 3 & 4 & 2 & 1 & 5 & 11 & 2 & 28 \\
\hline- & 4 & 4 & 2 & 1 & 0 & 0 & 1 & 12 \\
\hline \multicolumn{9}{|c|}{ Astler-Coller } \\
\hline & & & B 1 & B 2 & $\mathrm{C1}$ & $\mathrm{C2}$ & D & Total \\
\hline+ & & & 3 & 6 & 2 & 15 & 2 & $28 *$ \\
\hline- & & & 4 & 6 & 1 & 0 & 1 & 12 \\
\hline
\end{tabular}

* Existe associação significativa $(p=0.0244)$. 
Tabela 4 - Associação entre o estadiamento segundo sistema TNM e Astler-Coller no adenocarcinoma de intestino e a expressão da proteína Cox-2.

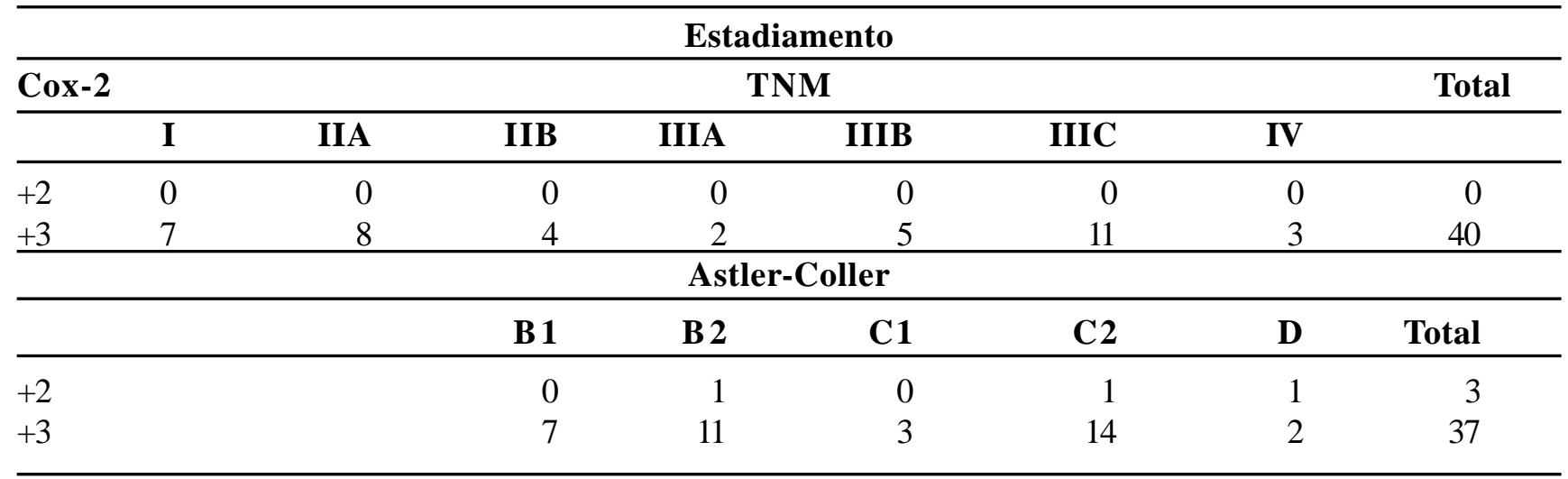

de tratamentos complementares ${ }^{20}$. A ressecção cirúrgica continua sendo a modalidade terapêutica mais efetiva para o tratamento desta neoplasia em fases iniciais. Em contraste, nos pacientes em que a cirurgia não é o tratamento de escolha, é fundamental a utilização de fatores prognósticos para melhor compreender a história natural do tumor e definir a melhor terapia ${ }^{21}$.

No nosso estudo, a população avaliada tinha entre 61 a 80 anos, sendo a idade média 61,8 anos, semelhante ao encontrado na literatura ${ }^{22,23}$, mostrando prevalência da doença em pessoas acima de 50 anos. $\mathrm{O}$ adenocarcinoma foi significativamente mais freqüente no cólon descendente ( $95 \%$ dos casos), semelhante ao relatado anteriormente ${ }^{22}$.

A marcação imuno-histoquímica foi maior, de uma maneira geral, em casos III-C uma vez que $100 \%$ dos III-C foram positivos para p53 e para Cox-2 (9\% positivo $2+$ e $91 \%$ positivo $3+$ ). Além disso, $100 \%$ dos casos III-B foram positivos para p53 e Cox-2 3+. Este fato é concordante com estudos que relacionam p53 e Cox-2 com pior prognóstico ${ }^{1,12,24,25}$. Neste estudo foi possível observar que proporcionalmente à progressão do estadiamento houve aumento da expressão destes marcadores, apesar destes dados não demonstrarem diferença significativa.

Quando fizemos a associação entre os marcadores e a classificação segundo Astler-Coller, observamos uma prevalência na classificação C2 . Destes, $100 \%$ foram positivos para p53 e Cox-2
(93,3 \% Cox-2 positivo $3+$ e $6,6 \%$ Cox -2 positivo $2+)$. Esta observação parece estar relacionada a um pior prognóstico na medida em que a expressão da p53 e Cox-2 aumentam de acordo com a imaturidade das células tumorais. Estes achados são concordantes com vários estudos encontrados na literatu$\mathrm{ra}^{1,9,12,18,24,26,27,28,29,30,31,32}$. Destaca-se que em estágio mais adiantado, estágio $\mathrm{D}$ de Dukes e $\mathrm{D}$ de Astler-Coller, foi observado um discreto decréscimo na marcação p53, enquanto a marcação Cox-2 se manteve com $100 \%$ de positividade.

$\mathrm{Na}$ maioria das associações realizadas houve concordância de expressão entre p53 e Cox-2, sugerindo mais estudos que abordem a expressão da Cox 2 e da p53 como fatores interligados. Tais achados encontram concordância na literatura, uma vez que vários oncogenes, fatores de crescimento e promotores de tumor podem apresentar a capacidade de induzir a expressão de Cox-2 em células malignas. Além disso, o p53 selvagem é capaz de suprimir a transcrição do Cox2, sugerindo que o p53 é, também, um determinante para a expressão do Cox-2. Este, por sua vez, pode gerar efeitos na proliferação celular e no prognóstico, aumentando o potencial metastático de células malignas $^{1,10}$.

Embora estes achados não possam suportar a hipótese de associação entre os marcadores p 53 e Cox2 com o estadiamento do tumor de intestino, sua contribuição é relevante para futuros estudos por apontar relações de extrema importância em torno destes marcadores como fatores prognósticos. 
Rev bras Coloproct Janeiro/Março, 2008
Expressão das Proteínas p53 e Cox-2 em

Adenocarcinoma Intestinal e Mucosa Adjacente Carlos Roberto Felin e Cols.
Vol. 28

ABSTRACT: The objective of this study is to establish a possible association between staging and the expression of p53 and cyclooxygenase-2 (Cox-2) proteins in intestinal adenocarcinoma. A retrospective study of 40 embedded paraffins with surgical specimens of intestinal Adenocarcinoma was performed. The samples were collected during 1998 and 2003 at Hospital Universitário de Santa Maria, RS, Brasil. Samples of non-tumoral tissues $(n=40)$ were used as a negative control. The expression of p53 and Cox-2 was immunohistochemically studied. The expression of p53 and Cox-2 proteins was significantly higher in the tumor material when compared to the normal adjacent mucosa. Cox-2 expression was found in $100 \%$ of tumoral cases, while p53 expression was noted in 70\% of tumor blocks. There is significantly association between the expression of p53 protein and AstlerColler staging, but no with TNM-stage. There was no significant correlation between Cox-2 expression and intestinal adenocarcinoma staging. Our findings suggest that the expression of p53 and Cox-2 proteins could have association with AstlerColler staging.

Key words: Intestinal adenocarcinoma. p53 protein. Cox-2 protein. Immunohistochemistry. Tumor staging.

\section{REFERÊNCIAS}

1. Guimarães JR. Manual de oncologia. 2nd ed. São Paulo: Libbs Farmacêutica, 2006. p.461-72.

2. Brentani MM, Coelho FRG, Kowalski LP. Bases da Oncologia. 2nd ed. São Paulo: Lemor, 2003. p.136-45.

3. Cotran RS, Kumar V, Collins T. Robbins Patologia estrutural e funcional. 7th ed. Rio de Janeiro: Guanabara Koogan, 2006. p.281-356;899-912.

4. Sternberg S, Antonioli D, Carter D, Mills S, Oberman H. Diagnostic surgical pathology. 3rd ed. Philadelphia: Lippincott Williams \& Wilkins, 1999. p.1424-67.

5. Sobin LH, wittekind $\mathrm{CH}$ (ed). Classification of Malignant Tumours. International Union Against Cancer. 6th ed. WhileyLiss, 2004. p.77-81.

6. Alves VFA, Bacchi CE, Vassallo J. Manual de ImunoHistoquímica. 1 st ed. São Paulo: Sociedade Brasileira de Patologia, 1999. p.237-57.

7. Chen WC, Lin MS, Zhang BF, Fang, J, Zhou Q, Hu Y, et al. Survey of molecular profiling during human colon cancer development and progression by immunohistochemical staining on tissue microarray. World J Gastroenterol 2007; 13: 699708.

8. Hsu SM, Raine L, fanger $\mathrm{H}$. Use of avidin-biotin peroxidase complex $(\mathrm{ABC})$ in immunoperoxidase techniques: a comparison between $\mathrm{ABC}$ and unlabelled antibody (PAP) procedures. J Histochem Cytochem 1981; 29:577-80.

9. Kahlenberg MS, Stoler DL, Rodriguez-Bigas MA, Weber TK, Driscoll DL, Anderson GR, et al.p53 tumor suppressor gene mutations predict decreased survival of patients with sporadic colorectal carcinoma. Cancer 2000; 88:1814-9.

10. Ferreira CG, Rocha JC. Oncologia Molecular. 1st ed. São Paulo: Atheneu, 2004. p.171-81.

11. Louro ID, Llerena JC, Mello MS, Prolla P, Froes N. Genética Molecular do Câncer. 2nd ed. São Paulo: MSG, 2002. p.6390.

12. Pinho MSL. Biologia molecular do câncer: fundamentos para a prática médica. 1st ed. Rio de Janeiro: Revinter, 2005. p.83-4.
13. Simon SD. A biologia molecular aplicada ao diagnóstico e tratamento do câncer. Diálogo Científico 2004; 1(3):18-22.

14. Arao J, Sano Y, Fujii T, Kato S, Yoshino T, Ochiai A, et al. Cyclooxygenase-2 Is Overexpressed in Serrated Adenoma of the Colorectum. Dis Colon Rectum 2000; 44:1319-23.

15. Chapple KS, Scott N, Guillou PJ, Coletta PL, Hull MA. Analysis of Cyclooxigenase Expression in Human Colorectal Adenomas. Dis Colon Rectum 2002; 45:1316-24.

16. Cotti GCC, Santos FPS, Sebastianes FM, Habr-Gama A, Seid VE, Martino RB. Genética do câncer colorretal. Rev Med. (São Paulo) 2000; 79:45-64.

17. Riddell RH. ADASP Recommendations for the reporting of resected large intestinal carcinomas. With commentary. Association of Directors of Anatomic and Surgical Pathology. Pathol Case Rev 1998; 3:217-22.

18. Elsaleh H, Soontrapornchai P, Grieu F, Joseph D, Iacopetta B. P53 alterations have no prognostic or predictive significance in Dukes' C rectal carcinomas. Int J Oncol 1999; 15:1239-43.

19. Asano TK, Mcleod RS. Nonsteroidal anti-inflammatory drug and aspirin for the prevention of colorectal adenomas and cancer: a systematic review. Dis Colon Rectum 2004; 47:665$73,2004$.

20. Michelassi F, Vanucci L, Ayala JJ, Chappel R, Goldreg R, Block GE. Local recurrence after curative resection of colorectal adenocarcinoma. Surgery 1990; 108: 787-93.

21. Sather HN. The use of prognostic factors in clinical trials. Cancer 1986; 58: 461-7.

22. Durante AP, Bromberg SH, Barreto E, Cappellano G, de Godoy AC. Prognostic value of lymphatic vessel and neural invasion in colorectal carcinoma. Rev Assoc Med Bras 2004; 50: 21-6.

23. Ponz de Leon M, Marino M, Benatti P, Rossi G, Menigatti M, Pedroni M, et al. Trend of incidence, subsite distribution and staging of colorectal neoplasms in the 15-year experience of a specialised cancer registry. Ann Oncol 2004; 15: 940-6.

24. Cressey R, Pimpa S, Tontrong W, Watananupong O, Leartprasertsuke N. Expression of cyclooxygenase-2 colorectal adenocarcinoma is associated with p53 accumulation and hdm2 overexpression. Cancer Lett 2006; 233: 232-9. 
25. Pinho MSL. Proteína p53: algum valor clínico ou apenas pesquisa? Uma revisão da literatura. Rev Bras Coloproct 2000; 20: 258-260.

26. Bosari S, Viale G, Bossi P, Maggioni M, Coggi G, Murray JJ, et al. Cytoplasmic Accumulation of p53 Protein: an Independent Prognostic Indicator in Colorectal Adenocarcinomas. J Nat Cancer Inst 1994; 86:681-7.

27. Bouzourene H, Gervaz P, Cerottini JP, Benhattar J, Chaubert P, Saraga E, et al. p53 and Ki-ras as prognostic factors for Dukes' stage B colorectal cancer. Eur J Cancer 2000; 6:100815.

28. Gallego MG, Acenero MJ, Ortega S, Delgado AA, Cantero JL. Prognostic influence of p53 nuclear overexpression in colorectal carcinoma. Dis Colon Rectum 2000; 43:971-5.

29. Liu Y, Bodmer WF. Analysis of p53 mutations and their expression in 56 coloretal cancer cell lines. Proc Nat Acad Sci 2006; 103:976-81.

30. Russo A, Bazan V, Iacopetta B, Kerr D, Soussi T, Gebbia N, et al. The TP53 Colorectal Cancer International Collaborative
Study on the Prognostic and Predictive Significance of p53 Mutation: Influence of Tumor Site, Type of Mutation, and Adjuvant Treatment. J Clin Oncol 2005; 23:7518-28.

31. Shiota G, Ishida M, Noguchi N, Oyama K, Takano Y, Okubo $\mathrm{M}$, et al. Circulating p53 antibody in patients with colorectal cancer: relation to clinicopathologic features and survival. Dig Dis Sci 2000; 45:122-8.

32. Zhang $\mathrm{H}$. Evaluation of four antibodies in detecting $\mathrm{p} 53$ protein for predicting clinicopathological and prognostic significance in colorectal adenocarcinoma. Clin Cancer Res 1999; 5:4126-32.

\section{Endereço para Correspondência:} IVANA GRIVICICH

Laboratório de Marcadores de Estresse Celular, Centro de Pesquisas em Ciências Médicas, Universidade Luterana do Brasil Av Farroupilha, 8001, Prédio 22, $5^{\circ}$ andar, Canoas, RS, Brasil CEP: $92245-900$

Tel: +55-51-34779219

E-mail: grivicich@terra.com.br 